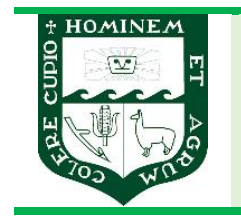

\title{
APTITUD COMBINATORIA DE LA VARIEDAD DE MAÍZ AMILÁCEO (Zea mays L.) PMD-638
}

\section{Combining ability of the maize (Zea mays L.) variety PMD-638}

\author{
Julián Chura Chuquija ${ }^{1 *}$ (D); Gilberto García Pando ${ }^{1}$ (D); Ricardo Sevilla Panizo1 (D) \\ ${ }^{1}$ Facultad de Agronomía, Programa de Investigación y Proyección Social en Maíz, \\ Universidad Nacional Agraria La Molina, 15024, Lima, Perú. \\ *E-mail: chura@1amolina.edu.pe
}

Recibido: 06/05/2019; Aceptado: 01/11/2021; Publicado: 31/12/2021

\begin{abstract}
The main objective was to estimate the effects of general combinatory ability (GCA) and specific (SCA) of ten $\mathrm{S}_{1}$ lines from PMD-638 variety. Experiment was carried out at IRD-Sierra, placed at Jauja locality during the 20162017 growing season. The field experimental design was Randomized Complete Blocks, and the variables of the study were grain yield (GY), male (MF) and female flowering (FF), plant (PH) and corn cob height (CH). GCA y SCA showed significant effects on the whole variables, except in MF in GCA. In GY, line 9 had the highest positive effect $(0,66)$ and line 1 the highest negative effect $(-0,68)$ and in SCA the 5x1 cross had the highest negative effect $(-4,33)$. In days to MF, line 9 had the higher effect for GCA $(2,44)$ and, in SCA, the higher effect was on the 6x5 cross $(-9,95)$. In days to FF the higher effect on GCA occurred on line $5(1,76) \mathrm{y}$, on SCA, the $7 \times 3$ cross $(4,82)$. Related to $\mathrm{PH}$ y $\mathrm{CH}$, the higher effect on GCA were on lines $9(-6,83$ y 6,36) y and $10(-10.11$ y 7.20$)$, respectively. On SCA, the 9x7 $(-21,65)$ y 9x5 $(20,66)$ crosses had higher values. On CH the 9x5 $(22,40)$ y $7 \times 3(19,65)$ crosses were found higher values.
\end{abstract}

Keywords. Maize | combinatory ability | additive effects

\section{RESUMEN}

El objetivo fue estimar los efectos de la aptitud combinatoria general (ACG) y específica (ACE) de diez líneas $\mathrm{S}_{1}$ provenientes de la variedad PMD-638. El experimento fue conducido en la localidad de Jauja IRD-Sierra en la campaña 2016-2017. El diseño experimental fue bloques completos al azar, las variables estudiadas fueron rendimiento de grano (RG), floración masculina (FM) y femenina (FF), altura de planta (AP) y mazorca (AM). La ACG y ACE presentaron efectos significativos en todas las variables a excepción de FM en ACG. En RG la línea 9 presentó mayor efecto positivo con 0,66 y la línea 1 el mayor efecto negativo con -0,68, y en ACE la cruza 5x1 presentó mayor efecto negativo con -4,33. En días a FM la línea 9 presentó el mayor efecto para la ACG con 2,44 y en ACE mayor efecto presentó la cruza 6x5 con -9,95, En días a FF se presentó mayor efecto de ACG con la línea 
$5(1,76)$ y en ACE la cruza 7x3 $(4,82)$. En cuanto a AP y AM se tuvo mayor efecto de ACG con las líneas 9 y 10 con los siguientes valores $-6,83$ y 6,36 (AP) y -10.11 y 7.20 (AM). En la ACE para AP destacaron las cruzas 9x7 $(-21,65)$ y $9 \times 5(20,66)$. En AM destacaron las cruzas 9x5 $(22,40)$ y $7 x 3(19,65)$.

Palabras clave: maíz amiláceo | aptitud combinatoria | efectos aditivos

\section{Forma de citar el artículo (Formato APA):}

Chura, J., García, G., \& Sevilla, R. (2021). Aptitud combinatoria de la variedad de maíz (Zea mays L.) amiláceo PMD-638. Anales Científicos. 81(2), 180-187. http://dx.doi.org/10.21704/ac.v82i2.1762

Autor de correspondencia (*): Julián Chura. Email: chura@lamolina.edu.pe

(C) Los autores. Publicado por la Universidad Nacional Agraria La Molina.

This is an open access article under the CC BY

\section{INTRODUCCIÓN}

El maíz amiláceo (Zea mays L.) es sembrado en las diferentes zonas altitudinales altoandinas del Perú, como un cultivo importante para la alimentación del poblador andino, por sus diversos usos en el consumo, principalmente, como choclo y grano. Hay una gran demanda en la zona de la costa como choclo principalmente para abastecer los supermercados y genera una alta rentabilidad para el agricultor andino, cuando lo comercializa. Sin embargo, el principal problema que afrontan es que no logran producir una mayor cantidad de choclos de primera, que es demandado por los pobladores de la costa, debido a que utilizan solo variedades mejoradas y una alternativa es generar híbridos que facilita una mayor producción de choclo de primera, debido a que es más uniforme que una variedad en sus características.

Uno de los principales objetivos del Programa de Investigación y Proyección Social en Maíz de la Universidad Nacional Agraria La Molina ha sido determinar líneas endogámicas con las cuales se puedan formar híbridos de buenas condiciones agronómicas y de altos rendimientos para grano y choclo. Para ello, el conocimiento del tipo de acción génica es un aspecto muy importante en establecer por el fitomejorador, principalmente en la determinación del método de mejoramiento más adecuado, con el fin de maximizar el tiempo y la eficiencia para obtener una variedad mejorada (Wong et al., 2007). La acción génica aditiva está muy relacionada con la ACG y la acción génica no aditiva con la ACE (Inram et al., 2004; Hallauer et al., 2010).
La aptitud combinatoria general es definida como el comportamiento promedio de una línea es sus combinaciones híbridas y la aptitud combinatoria específica como aquellos casos en que ciertas combinaciones se comportan relativamente mejor o peor de lo que podría esperarse en base al comportamiento promedio de las líneas involucradas (Sprague y Tatum, 1942).

El estudio de la aptitud combinatoria es muy importante debido a que va a permitir la identificación de los progenitores potenciales, que pueden ser muy útiles para formar híbridos con alto potencial de rendimiento o para el desarrollo de nuevas poblaciones compuestas o sintéticos (Martínez, 1983).

Las cruzas simples de alta $\mathrm{x}$ baja ACG resultaron más rendidoras que las de alta $\mathrm{x}$ alta y baja $\mathrm{x}$ baja, según Vélez-Torres et al. (2018). Las cruzas simples de alta $\mathrm{x}$ alta ACG mostraron la mayor estabilidad debido a la alta ACG de ambas líneas y a un alto grado de endogamia, que permitieron que dichos genotipos conservaran los efectos aditivos a través de ambientes, pero con rendimiento por debajo de la media.

Mendes et al. (2003), Reyes et al. (2004) y Escorcia et al. (2010) mencionan que una cruza simple es de alto rendimiento de grano si las dos líneas son de alta ACG, o al menos una línea es de alta ACG, y de efectos positivos la ACE.

El presente trabajo de investigación tuvo como objetivo estimar los efectos de la aptitud combinatoria general y específica en la variedad de maíz amiláceo PMD-638. 


\section{MATERIALES Y MÉTODOS}

En este trabajo se utilizaron diez líneas de maíces blancos amiláceos (Tabla 1) con nivel de endogamia $S_{1}$ derivadas de la variedad PMS-638, las cuales fueron seleccionados con un probador identificado como la variedad Blanco Urubamba.

Tabla 1. Líneas de maíz (Zea mays L.) amiláceo derivadas de la variedad PMD-638.

\begin{tabular}{|c|c|}
\hline Número de línea & Línea \\
\hline 1 & PMD - $638-S 1-107$ \\
\hline 2 & PMD - $638-\mathrm{S} 1-110$ \\
\hline 3 & PMD - $638-\mathrm{S} 1-129$ \\
\hline 4 & PMD - $638-\mathrm{S} 1-143$ \\
\hline 5 & PMD - $638-\mathrm{S} 1-146$ \\
\hline 6 & PMD - $638-\mathrm{S} 1-226$ \\
\hline 7 & PMD - $638-\mathrm{S} 1-236$ \\
\hline 8 & PMD - $638-\mathrm{S} 1-275$ \\
\hline 9 & PMD - $638-\mathrm{S} 1-459$ \\
\hline 10 & PMD - $638-\mathrm{S} 1-129$ \\
\hline
\end{tabular}

Las cruzas se realizaron en el periodo 2015 - 2016 en el Instituto Regional de Desarrollo (IRD) de Sierra localizado en Jauja y la $F_{1}$ evaluada en la campaña 2016-2017 en el mismo lugar, mediante un diseño de bloques completo al azar con cuatro repeticiones. La parcela experimental estuvo constituida por un surco de $4 \mathrm{~m}$ de largo, con 11 golpes por surco, sembrándose tres semillas por golpe y se dejaron 2 plantas por golpe al desahíje y distanciados a 0,40 m entre golpes y $0,80 \mathrm{~m}$ entre surcos. con una densidad de 62500 plantas/ha.

Las variables evaluadas fueron: días a floración masculina (FM), días a floración femenina (FF), altura de planta (AP), altura de mazorca (AM) y rendimiento de grano (RG), el rendimiento de grano se llevó al 14\% de humedad, se hizo la corrección por fallas y se llevó a t/ha.

El análisis genético se realizó de acuerdo a la metodología propuesta por Griffing (1956) método IV, modelo I adaptado para múltiples años, donde;

$$
\mathrm{Y}_{\mathrm{ijk}}=\mathrm{u}+\mathrm{G}_{\mathrm{i}}+\mathrm{G}_{\mathrm{j}}+\mathrm{S}_{\mathrm{ij}}+\beta_{\mathrm{k}}+\varepsilon_{\mathrm{ijk}} \text {, siendo: }
$$

$\mathrm{Y}_{\mathrm{ijk}}=$ valor medio del híbrido $\mathrm{ij}(\mathrm{i}, \mathrm{j}=1,2 \ldots \mathrm{p}, \mathrm{i}<\mathrm{j})$ en el k-ésimo bloque; $\mathrm{u}=$ media general;

$\mathrm{G}_{\mathrm{i}}, \mathrm{G}_{\mathrm{j}}=$ efecto de la habilidad combinatoria general del i-ésimo y j-ésimo progenitor, respectivamente;

$S_{\mathrm{ij}}=$ efecto de la habilidad combinatoria específica para los cruzamientos entre los progenitores del orden i y j;

$\beta_{\mathrm{k}}=$ efecto del k-ésimo bloque;

$\varepsilon_{\mathrm{kij}}=$ error experimental.

Los efectos de la ACG de cada parental fueron estimados de la siguiente manera:

gi=yi.-y..

$\mathrm{gj}=\mathrm{y} . \mathrm{j}-\mathrm{y}$.

Para los efectos de la ACE fue estimada de la siguiente manera:

Sij=yij-yi.-y.j+y.

Dónde:

gi: es el efecto HCG del i-ésimo genotipo parental gj: es el efecto HCG del j-ésimo genotipo parental

Sij: es la HCE vigente para el cruce entre el i-ésimo y jésimo genotipo parental

yij: Promedio observado del resultado de la cruza entre el i-ésimo genotipo al j-ésimo genotipo.

yi.: Promedio observado de todos los genotipos donde interviene el i-ésimo genotipo parental.

y.j: Promedio observado de todos los genotipos donde interviene el j-ésimo genotipo parental.

y..: Promedio observado de todos los genotipos resultantes de las cruzas entre parentales.

\section{RESULTADOS Y DISCUSIÓN}

En la fuente de variación mostrada en la (Tabla 2) se encontró alta significación estadística para Cruzas, ACG y ACE en todas las variables estudiadas; a excepción de la variable días a FM en donde se encontró significación estadística para cruzas y ACE, no se encontró significación estadística para ACG. La ACG y ACE en las variables estudiadas nos indica la variabilidad en los efectos génicos aditivos y dominantes, similar resultado obtuvo en maíces blancos (Cervantes-Ortiz et al., 2018), a excepción de la variable floración masculina donde fue más importante los efectos génicos no aditivos; el conocimiento de estos efectos génicos es muy importantes para el método de mejoramiento que va seguir el mejorador para obtener una variedad mejorada de buenas características que desea desarrollar. 
En la (Tabla 3) observamos en rendimiento de grano (t/ha) en donde destacaron las cruzas 8x6 (15,70), 9x3 $(15,25)$ y 9x5 (15,08), 6x1 (14.8), 8x5 (14.74), 9x1 (14.49), 7x3 (14.21), 7x6 (13.9), 10x7 (13.62), 10x9 (13.58) y son similares estadísticamente a una probabilidad de 0.05 ; estas combinaciones tuvieron alta y baja ACG concordando con lo indicado por (VélezTorres et al., 2018; Acevedo-Cortés et al., 2020). El alto rendimiento de grano está muy relacionado con plantas altas y tardías. Días a floración masculina varió de 108 días a 89 días con las cruzas 9x5 y 6x5 respectivamente, días a floración femenina varió de 114 días a 103 días con las cruzas 9x5 y 10x3 respectivamente. En altura de planta la cruza 9x5 presentó la mayor altura con $224 \mathrm{~cm}$ y la menor altura con la cruza $6 x 3$ con $172 \mathrm{~cm}$ y en altura de mazorca la cruza $7 \times 3$ presentó la mayor altura de mazorca y la menor altura de mazorca lo presentó la cruza $5 \times 4$ con $73 \mathrm{~cm}$, la cruza de mayor rendimiento $8 \times 6$ obtuvo una altura de planta de $204 \mathrm{~cm}$ y una altura de mazorca de $101 \mathrm{~cm}$ que difiere estadísticamente a una probabilidad de 0.05 de las plantas que presentaron mayor altura de planta y mazorca.

En la (Tabla 4) se presenta los efectos de la ACG y en la (Tabla 5) los efectos de la ACE. En rendimiento de grano, las líneas 9 y 8 presentaron mayores efectos positivos $(0,66,0,42$, respectivamente) y las líneas 1,4 y 10 los mayores efectos negativos $(-0,68,-0,53$ y $-0,50$, respectivamente). En ACE las cruzas 6x1, 8x6, 9x1, 9x3, 10x7 y 9x5 presentaron los mayores efectos positivos $(2.97,2.78,2.29,2.08,1.94$ y 1.91 respectivamente) y las cruzas $5 \times 1,8 \times 7$ y $9 \times 6$ presentaron mayores efectos negativos $(-4,33,-3,38$ y 3,04 , respectivamente). En días a floración masculina, el mayor efecto para la ACG fue encontrado en la línea $9(2,44)$ y en el ACE el mayor efecto ocurrió en la cruza $6 \times 5(-9,95)$. En días a floración femenina se presentó mayor efecto de ACG con la línea $5(1,76)$ y en ACE la cruza $7 x 3(4,82)$. En altura de planta y altura de mazorca se tuvo mayor efecto de ACG con las líneas 9 (-6,83 y 10.11, respectivamente) y $10 \quad(6.36$ y 7.20 , respectivamente). En ACE para altura de planta destacaron las cruzas 9x7 $(-21,65)$ y 9x5 $(20,66)$, y para altura de mazorca destacaron las cruzas $9 \times 5(22,40)$ y $7 \times 3(19,65)$. Una cruza simple es de alto rendimiento si los progenitores presentan alta ACG o alta ACE.

Las combinaciones de las líneas con alto y bajo efecto en ACG presentaron los mayores rendimientos de grano, el cual concuerda con los hallazgos de (VélezTorres et al., 2018; Acevedo-Cortés et al., 2020).

Con las líneas 9, 8, 3, 5 y 6 que presentaron ACG de 0.66, 0.42, 0.29, 0.29 y 0.28 respectivamente positivos, se puede formar una variedad sintética debido a que las cruzas $8 \times 6,9 \times 3,9 \times 5$ presentaron los mayores rendimientos de grano y los que presentan mayores valores de ACE se pueden formar híbridos en un programa de mejoramiento lo cual concuerda con (Guillen- de la Cruz et al. 2009; Rodríguez-Pérez et al. 2019). Las cruzas de mayor rendimiento de grano se obtuvieron con líneas de mayor ACG tal como indican Rodríguez et al. (2020).

Tabla 2. Cuadrados medios del análisis de variancia dialélico para días a floración masculina, días a floración femenina, altura de planta $(\mathrm{cm})$, altura de mazorca $(\mathrm{cm})$ y rendimiento de grano (t/ha), Jauja 2016-2017.

\begin{tabular}{|c|c|c|c|c|c|c|c|c|c|c|c|}
\hline \multirow{2}{*}{$\begin{array}{l}\text { Fuente de } \\
\text { variación } \\
\text { Bloques }\end{array}$} & \multirow{2}{*}{$\begin{array}{l}\text { GL } \\
3\end{array}$} & \multicolumn{2}{|c|}{$\begin{array}{l}\text { Rendimiento } \\
\text { grano }\end{array}$} & \multicolumn{2}{|c|}{$\begin{array}{l}\text { Floración } \\
\text { masculina }\end{array}$} & \multicolumn{2}{|c|}{$\begin{array}{l}\text { Floración } \\
\text { femenina }\end{array}$} & \multicolumn{2}{|c|}{ Altura planta } & \multicolumn{2}{|l|}{$\begin{array}{l}\text { Altura } \\
\text { mazorca }\end{array}$} \\
\hline & & 3,25 & & 59,66 & & 52,11 & $* *$ & 1119,83 & $* *$ & 126,02 & \\
\hline Cruzas & 44 & 11,72 & $* *$ & 59,68 & $*$ & 24,53 & $* *$ & 535,54 & $* *$ & 609,50 & $* *$ \\
\hline ACG & 9 & 6,75 & $* *$ & 51,62 & & 35,98 & $* *$ & 690,91 & $* *$ & 1148,43 & $* *$ \\
\hline ACE & 35 & 12,99 & $* *$ & 61,76 & $*$ & 21,59 & $* *$ & 495,59 & $* *$ & 470,92 & $* *$ \\
\hline Error & 132 & 2,51 & & 37,76 & & 11,85 & & 130,69 & & 150,78 & \\
\hline Total & 179 & & & & & & & & & & \\
\hline C.V:(\%) & & 12,97 & & 6,34 & & 3,21 & & 5,93 & & 13,27 & \\
\hline Promedio & & 12,21 & & 96,92 & & 107,33 & & 192,63 & & 92,52 & \\
\hline
\end{tabular}

*** significación al $5 \%$ y $1 \%$ de probabilidad. 
Chura, J., García, G., \& Sevilla, R. (2021). Anales Científicos. 82(2), 180-187. DOI. 10.21704/ac.v82i2.1762

Tabla 3. Medias de las variables evaluadas rendimiento de grano (t/ha), días a floración masculina, días a floración femenina, altura de planta (cm), altura de mazorca (cm). Jauja 2016-2017.

\begin{tabular}{|c|c|c|c|c|c|}
\hline Cruzas & $\begin{array}{c}\text { Rendimiento } \\
\text { grano }\end{array}$ & $\begin{array}{l}\text { Floración } \\
\text { masculina }\end{array}$ & $\begin{array}{l}\text { Floración } \\
\text { femenina }\end{array}$ & $\begin{array}{c}\text { Altura de } \\
\text { planta }\end{array}$ & $\begin{array}{r}\text { Altura } \\
\text { mazorca }\end{array}$ \\
\hline $2 \times 1$ & 13,14 & 99,25 & 107,25 & 207,50 & 103,25 \\
\hline $3 \times 1$ & 11,05 & 94,75 & 105,00 & 200,50 & 105,50 \\
\hline $4 \times 1$ & 10,21 & 95,75 & 105,75 & 187,25 & 102,25 \\
\hline $5 \times 1$ & 7,51 & 92,75 & 105,00 & 188,25 & 87,25 \\
\hline $6 \times 1$ & 14,80 & 100,00 & 108,25 & 198,00 & 105,75 \\
\hline $7 \times 1$ & 11,05 & 98,25 & 107,25 & 207,75 & 99,75 \\
\hline $8 \times 1$ & 11,65 & 96,75 & 106,50 & 191,75 & 92,00 \\
\hline $9 \times 1$ & 14,49 & 97,00 & 107,75 & 212,25 & 115,75 \\
\hline $10 \times 1$ & 10,63 & 95,00 & 106,50 & 180,50 & 77,25 \\
\hline $3 \times 2$ & 11,53 & 95,00 & 105,00 & 185,75 & 93,00 \\
\hline $4 \times 2$ & 10,70 & 95,75 & 104,75 & 192,25 & 88,50 \\
\hline $5 \times 2$ & 13,23 & 99,25 & 111,00 & 204,25 & 109,75 \\
\hline $6 \times 2$ & 11,14 & 96,25 & 107,50 & 194,00 & 86,75 \\
\hline $7 \times 2$ & 13,29 & 103,00 & 109,00 & 202,75 & 107,00 \\
\hline $8 \times 2$ & 12,46 & 97,75 & 106,50 & 182,50 & 81,50 \\
\hline $9 \times 2$ & 12,20 & 95,00 & 106,50 & 186,25 & 83,25 \\
\hline $10 \times 2$ & 10,55 & 94,75 & 104,75 & 181,75 & 89,25 \\
\hline $4 \times 3$ & 12,76 & 92,50 & 104,00 & 174,50 & 86,25 \\
\hline $5 \times 3$ & 13,11 & 97,25 & 106,50 & 188,00 & 84,75 \\
\hline $6 \times 3$ & 11,54 & 98,50 & 107,50 & 172,25 & 86,75 \\
\hline $7 \times 3$ & 14,21 & 94,50 & 111,75 & 184,50 & 121,00 \\
\hline $8 \times 3$ & 12,51 & 94,00 & 105,75 & 189,50 & 98,75 \\
\hline $9 \times 3$ & 15,25 & 104,50 & 110,00 & 211,25 & 118,00 \\
\hline $10 \times 3$ & 10,28 & 93,25 & 103,00 & 178,75 & 81,25 \\
\hline $5 \times 4$ & 11,82 & 96,25 & 107,75 & 183,50 & 73,00 \\
\hline $6 \times 4$ & 10,87 & 89,25 & 106,50 & 178,75 & 74,00 \\
\hline $7 \times 4$ & 12,62 & 96,25 & 105,75 & 196,00 & 89,00 \\
\hline $8 \times 4$ & 13,31 & 98,50 & 107,25 & 198,25 & 92,50 \\
\hline $9 \times 4$ & 11,58 & 101,25 & 111,00 & 195,25 & 88,25 \\
\hline $10 \times 4$ & 11,85 & 95,00 & 106,25 & 189,25 & 78,75 \\
\hline $6 \times 5$ & 13,06 & 88,50 & 107,00 & 208,25 & 98,00 \\
\hline $7 \times 5$ & 10,97 & 101,25 & 108,50 & 190,25 & 87,75 \\
\hline $8 \times 5$ & 14,74 & 101,25 & 109,00 & 194,50 & 83,75 \\
\hline $9 \times 5$ & 15,08 & 107,50 & 114,25 & 223,75 & 120,75 \\
\hline $10 \times 5$ & 12,77 & 100,00 & 111,00 & 185,75 & 76,75 \\
\hline $7 x 6$ & 13,90 & 101,75 & 111,00 & 204,75 & 99,25 \\
\hline $8 \times 6$ & 15,70 & 101,50 & 112,75 & 203,50 & 101,00 \\
\hline $9 x 6$ & 10,12 & 100,25 & 106,50 & 181,25 & 90,50 \\
\hline $10 \times 6$ & 11,08 & 96,75 & 103,25 & 192,25 & 82,75 \\
\hline $8 \times 7$ & 9,22 & 92,75 & 105,75 & 182,00 & 77,00 \\
\hline $9 x 7$ & 10,78 & 92,00 & 104,75 & 179,50 & 87,75 \\
\hline $10 \times 7$ & 13,62 & 91,25 & 106,50 & 203,50 & 92,25 \\
\hline $9 \times 8$ & 12,13 & 96,50 & 108,50 & 208,75 & 92,25 \\
\hline $10 \times 8$ & 11,58 & 95,00 & 105,75 & 181,00 & 79,75 \\
\hline $10 \times 9$ & 13,58 & 97,75 & 108,00 & 186,25 & 93,75 \\
\hline DLS 0,05 & 2,22 & 8,60 & 4,81 & 15,99 & 17,18 \\
\hline DLS 0,01 & 2,93 & 11,36 & 6,36 & 21,13 & 22,69 \\
\hline
\end{tabular}


Tabla 4. Efectos de aptitud combinatoria general (ACG) de diez líneas de maíz amiláceo para rendimiento de grano (t/ha), días a floración masculina, días a floración femenina, altura de planta $(\mathrm{cm})$, altura de mazorca $(\mathrm{cm})$. Jauja 2016-2017.

\begin{tabular}{crrrrr}
\hline Líneas & Rendimiento grano & Floración masculina & Floración femenina & Altura de planta & Altura mazorca \\
\hline 1 & $-0,68$ & $-0,34$ & $-0,84$ & 5,01 & 7,01 \\
2 & $-0,21$ & 0,47 & $-0,46$ & 0,42 & 1,20 \\
3 & 0,29 & $-1,00$ & $-0,93$ & $-6,08$ & 5,33 \\
4 & $-0,53$ & $-1,47$ & $-0,87$ & $-4,83$ & $-7,52$ \\
5 & 0,29 & 1,47 & 1,76 & 4,11 & $-1,36$ \\
6 & 0,28 & 0,06 & 0,54 & $-0,08$ & $-0,99$ \\
7 & $-0,03$ & $-0,16$ & 0,54 & 2,17 & 3,51 \\
8 & 0,42 & 0,22 & 0,23 & $-0,24$ & $-4,27$ \\
9 & 0,66 & 2,44 & 1,41 & 6,36 & 7,20 \\
10 & $-0,50$ & $-1,69$ & $-1,37$ & $-6,83$ & $-10,11$ \\
\hline
\end{tabular}

Tabla 5. Efectos de aptitud combinatoria específica (ACE) de nueve líneas de maíz para rendimiento de grano (t/ha), días a floración masculina, días a floración femenina, altura de planta (cm), altura de mazorca (cm). Jauja 20162017.

\begin{tabular}{|c|c|c|c|c|c|}
\hline Cruzas & Rendimiento grano & Floración masculina & Floración femenina & Altura de planta & Altura mazorca \\
\hline $2 \times 1$ & 1,81 & 2,21 & 1,22 & 9,44 & 2,52 \\
\hline $3 \times 1$ & $-0,78$ & $-0,82$ & $-0,56$ & 8,94 & 0,65 \\
\hline $4 \times 1$ & $-0,80$ & 0,65 & 0,13 & $-5,56$ & 10,24 \\
\hline $5 \times 1$ & $-4,33$ & $-5,29$ & $-3,25$ & $-13,50$ & $-10,92$ \\
\hline $6 \times 1$ & 2,97 & 3,36 & 1,22 & 0,44 & 7,21 \\
\hline $7 \mathrm{x} 1$ & $-0,46$ & 1,83 & 0,22 & 7,94 & $-3,29$ \\
\hline $8 \times 1$ & $-0,31$ & $-0,04$ & $-0,22$ & $-5,65$ & $-3,26$ \\
\hline $9 \times 1$ & 2,29 & $-2,01$ & $-0,15$ & 8,25 & 9,02 \\
\hline $10 \times 1$ & $-0,41$ & 0,11 & 1,38 & $-10,31$ & $-12,17$ \\
\hline $3 \times 2$ & $-0,76$ & $-1,39$ & $-0,93$ & $-1,22$ & $-6,04$ \\
\hline $4 \times 2$ & $-0,78$ & $-0,17$ & $-1,25$ & 4,03 & 2,30 \\
\hline $5 \times 2$ & 0,94 & 0,40 & 2,38 & 7,10 & 17,40 \\
\hline $6 \times 2$ & $-1,15$ & $-1,20$ & 0,10 & 1,03 & $-5,98$ \\
\hline $7 \times 2$ & 1,32 & 5,77 & 1,60 & 7,53 & 9,77 \\
\hline $8 \times 2$ & 0,04 & 0,15 & $-0,59$ & $-10,31$ & $-7,95$ \\
\hline $9 \times 2$ & $-0,46$ & $-4,82$ & $-1,78$ & $-13,15$ & $-17,67$ \\
\hline $10 \times 2$ & $-0,96$ & $-0,95$ & $-0,75$ & $-4,47$ & 5,65 \\
\hline $4 \times 3$ & 0,79 & $-1,95$ & $-1,53$ & $-7,22$ & $-4,07$ \\
\hline $5 \times 3$ & 0,31 & $-0,14$ & $-1,65$ & $-2,65$ & $-11,73$ \\
\hline $6 \times 3$ & $-1,25$ & 2,52 & 0,57 & $-14,22$ & $-10,10$ \\
\hline $7 \times 3$ & 1,74 & $-1,26$ & 4,82 & $-4,22$ & 19,65 \\
\hline $8 \times 3$ & $-0,41$ & $-2,14$ & $-0,87$ & 3,19 & 5,18 \\
\hline $9 \times 3$ & 2,08 & 6,15 & 2,19 & 18,35 & 12,96 \\
\hline $10 \times 3$ & $-1,72$ & $-0,98$ & $-2,03$ & $-0,97$ & $-6,48$ \\
\hline $5 \times 4$ & $-0,16$ & $-0,67$ & $-0,47$ & $-8,40$ & $-10,64$ \\
\hline $6 \times 4$ & $-1,10$ & $-6,26$ & $-0,50$ & $-8,97$ & $-10,01$ \\
\hline $7 \times 4$ & 0,97 & 0,96 & $-1,25$ & 6,03 & 0,49 \\
\hline $8 \times 4$ & 1,20 & 2,83 & 0,57 & 10,69 & 11,77 \\
\hline $9 \times 4$ & $-0,77$ & 3,36 & 3,13 & 1,10 & $-3,95$ \\
\hline $10 \times 4$ & 0,66 & 1,24 & 1,16 & 8,28 & 3,86 \\
\hline $6 \times 5$ & 0,26 & $-9,95$ & $-2,62$ & 11,60 & 7,83 \\
\hline $7 \times 5$ & $-1,51$ & 3,02 & $-1,12$ & $-8,65$ & $-6,92$ \\
\hline $8 \times 5$ & 1,81 & 2,65 & $-0,31$ & $-2,00$ & $-3,14$ \\
\hline $9 \times 5$ & 1,91 & 6,68 & 3,75 & 20,66 & 22,40 \\
\hline $10 \times 5$ & 0,76 & 3,30 & 3,28 & $-4,15$ & $-4,29$ \\
\hline $7 \times 6$ & 1,44 & 4,93 & 2,60 & 10,03 & 4,21 \\
\hline
\end{tabular}




\begin{tabular}{crrrrr}
$8 \times 6$ & 2,78 & 4,30 & 4,66 & 11,19 & 13,74 \\
$9 \times 6$ & $-3,04$ & 0,83 & $-2,78$ & $-17,65$ & $-8,23$ \\
$10 \times 6$ & 1,46 & $-3,25$ & 6,53 & 1,33 \\
$8 \times 7$ & $-0,92$ & $-4,23$ & $-2,34$ & $-12,56$ & $-14,76$ \\
$9 \times 7$ & $-3,38$ & $-7,20$ & $-4,53$ & $-21,65$ & $-15,48$ \\
$10 \times 7$ & $-2,06$ & $-3,82$ & 0,00 & 15,53 & 6,33 \\
$9 \times 8$ & 1,94 & $-3,07$ & $-0,47$ & 10,00 & $-3,20$ \\
$10 \times 8$ & $-1,16$ & $-0,45$ & $-0,43$ & $-4,56$ & 1,61 \\
$10 \times 9$ & $-0,55$ & 0,08 & 0,63 & $-5,90$ & 4,15 \\
\hline
\end{tabular}

\section{CONCLUSIONES}

Los efectos de la aptitud combinatoria general (efectos aditivos) fueron más importantes para días a floración femenina, altura de planta y mazorca; los efectos de la aptitud combinatoria especifican (efectos no aditivos) fueron más importantes para rendimiento de grano y días a floración masculina. La línea 9 presento mayor efecto para aptitud combinatoria general para las variables estudiadas. Las cruzas $8 \times 6,9 \times 3$ y $9 \times 5$ destacaron en rendimiento de grano, siendo tardíos y de porte alto y presentaron los mayores efectos de aptitud combinatoria específica positivos; la cruza $6 \times 5$ destacó en precocidad.

\section{Agradecimiento}

Los autores desean agradecer a la entidad financiera INOVATE Perú, anteriormente FINCYT, por haber financiado la ejecución del presente proyecto de investigación.

\section{Conflictos de intereses}

Los autores firmantes del presente trabajo de investigación declaran no tener ningún potencial conflicto de interés personal o económico con otras personas $\mathrm{u}$ organizaciones que puedan influir indebidamente con el presente manuscrito.

\section{Contribuciones de los autores}

Preparación y ejecución: JCH, GG; Desarrollo de la metodología: JCH, GG; Concepción y diseño: JCH; Edición del artículo: JCH; Supervisión del estudio: JCH, GG, RS.

\section{REFERENCIAS}

- Acevedo-Cortés, M., Castillo-Gutiérrez, A., Andrade-Rodríguez, M., Nuñez-Valdez, M., Perdomo-Roldan; F., \& Suárez-Rodríguez, R. 2020. Aptitud combinatoria y potencial agronómico de líneas de maíz con diferente nivel de endogamia. Acta Agrícola y Pecuaria, 6 (1).

- Cervantes-Ortiz, F., Hernández-Esparza, J., García-Rodríguez, J., Antonio Rangel-Lucio, J., Andrio-Enríquez, E., Mendoza-Elos, M., Rodríguez-Pérez, G., \& Rodríguez-Mercado, D. (2018). Aptitud combinatoria general y específica de caracteres agronómicos en líneas de maíz (Zea mays L.) de baja endogamia. Chilean J. Agric. Anim. Sci., ex Agro-Ciencia (2018), 34(1),33-42.

- Escorcia, G., Molina, G., Castillo, G. \& Mejía, C. (2010). Rendimiento, heterosis y depresión endogámica de cruzas simples de maíz. Rev. Fitotec. Mex. 33, 271-279.

- Griffing B. (1956). Concept of general and specific combining ability in relation to diallel crossing systems. Aust. J. Biol. Sci. 9,463-493.

- Guillen-de la Cruz, P., de la Cruz-Lázaro, E., Castañón-Nájera, G., Osorio-Osorio, R., BritoManzano, N.P., Lozano-del Río, A., \& LópezNoverola, U. (2009). Aptitud combinatoria general y específica de germoplasma tropical de maíz. Tropical and Subtropical Agroecosystems, 10(1),101-107.

- Hallauer A. R., Carena, M., \& Miranda, F. 2010. Quantitative Genetics in Maize Breeding. Springer-Verlag. New York Inc. 664 p.

- Inram, M.S., Nawaz, M. H., Mahmood, M., \& Munir, M. (2004). General and specific combining ability studies in maize diallel crosses. Int. J. Agric. Biol. 6, 856-859.

- Martínez, G. A. (1983). Diseños y análisis de 
experimentos de cruzas dialélicas. Colegio de Posgraduados. México $251 \mathrm{p}$.

- Mendes, A., Carlini-Garcia, A., Resende, S., Figueireido, S.A., Franco, G. \& Lopes, S. (2003). Combining ability of inbred lines of maize and stability of their respective single-crosses. Sci. Agric. 60, 83-89.

- Reyes, L., Molina, G., Oropeza, R., \& Moreno, P. (2004). Cruzas dialélicas entre líneas autofecundadas de maíz derivadas de la raza Tuxpeño. Rev. Fitotec. Mex. 27, 49-56.

- Rodríguez-Pérez, G., Zavala-García, F., TreviñoRamírez, J., Ojeda-Zacarías, C., Mendoza-Elos, M., Cervantes-Ortiz, F., Gámez-Vázquez, A., Andrio-Enríquez, E., \& Torres-Flores, J. (2019). Estimación de componentes genéticos en líneas endogámicas de maíz (Zea mays L.). Agrociencia, 53(2), 245-258.

- Rodríguez, G., Treviño, J. E.; Ojeda, M.; Cervantes, F., Avila, M. A., \& Gámez, A. J. (2020).
Parámetros genéticos y aptitud combinatoria de líneas de maíz para grano. Revista Mexicana Ciencias Agrícolas volumen 11 número 8.

- Sprague, G.F., \& Tatum, A. (1942). General vs. specific combining ability in single crosses of corn. J. Am. Soc. Agron. 34,923-932.

- Vélez-Torres, M., García-Zavala, J., Lobato-Ortiz, R., Benítez, I., López-Reynoso, J., MejíaContreras, J., \& Esquivel-Esquivel, G. (2018). Estabilidad del rendimiento de cruzas dialélicas entre líneas de maíz de alta y baja aptitud combinatoria general. Rev. Fitotec. Mex. 41 (2), 167-175.

- Wong, R.R., Gutiérrez, R.E., Palomo, E.G.A., Rodríguez, S., Córdoba, H., Espinoza, A.B. et al. (2007). Aptitud combinatoria de componentes del rendimiento en líneas de maíz para grano en la comarca lagunera, México. Rev. Fitotec. Mex. 30(2), 181-189. 